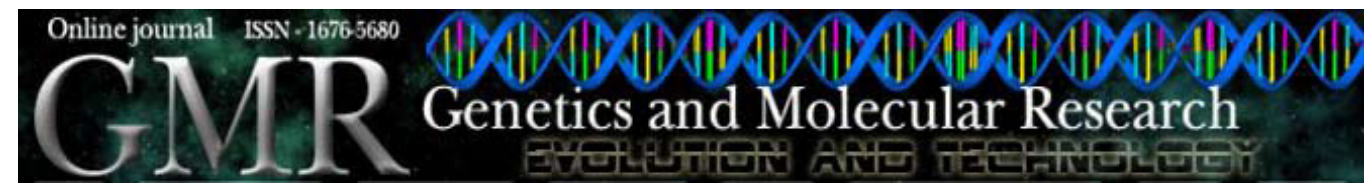

Letter to the Editor

\title{
Mitochondrial plasmid: could this be the next hit for genetic engineering?
}

\author{
S. Ijaz \\ Centre of Agricultural Biochemistry and Biotechnology, \\ University of Agriculture, Faisalabad, Pakistan \\ Corresponding author: S. Ijaz \\ E-mail: siddraijazkhan@yahoo.com
}

Genet. Mol. Res. 10 (1): 391-392 (2011)

Received October 4, 2010

Published March 1, 2011

DOI 10.4238/vol10-1gmr1092

Dear Sir,

In different biological systems autonomously replicated DNA molecules have been found with both terminal inverted repeats of variable length and protein covalently attached at the 5 ' ends (Turpen et al., 1987). These were formerly discovered in bacteria, but later similar molecules were found in eukaryotes. Another level of organization in plasmid-like DNA sporadically occurs in plant mitochondria and has been observed in a wide range of angiosperm (Esser et al., 1986). But in the chloroplasts of higher plants, there is no record of any plasmidlike DNA (Meinhardt et al., 1990).

Normally these extragenomic DNA molecules are found in the mitochondrial genome of fungi and plants and exhibit great diversity. Basically these are true plasmids, autonomously replicated elements that have slight or no homology to mitochondrial DNA. These small extragenomic DNA molecules (plasmids) reproduce inside living cells and replicate independently from genome, although some can integrate and replicate as a part of genomic DNA. Various types of mitochondrial plasmids have been found, e.g., circular DNA plasmids, linear DNA plasmids and RNA plasmids, and from total mitochondrial DNA these mitochondrial plasmids can easily be isolated and detected as extra-bands when DNA prepared from mitochondria is run on gel (Bengtsson and Andersson, 1997).

Replication of circular mitochondrial plasmids strongly resembles the mode of replication of bacterial plasmids. These modes were conventionally named sigma and theta. The location of priming sites for leading and lagging strand synthesis is very close to one another, 
within the origin of replication in the case of theta mode (Seegers et al., 1994), but different in the case of the sigma/rolling circle mode of replication. Antibiotic inhibiting mitochondrial protein synthesis resembles that of a prokaryotic system (Borst and Grivell, 1971) and these observations support the endosymbiotic theory and suggest that the ancestors of mitochondria are proteobacteria.

These characteristics show that these mitochondrial plasmids exhibit similarity to bacterial plasmids, so these plant mitochondrial plasmids can be used as vector/vehicles in DNA recombination technology instead of bacterial plasmids. And like chloroplast, mitochondrial genomes are also maternally inherited in most plant species and also have their own transcriptional translational machinery. Therefore, the mitochondrial genome can be used for transformation purposes with the certainty of transgene containment-like plastids.

If we use mitochondrial plasmids as the vector or vehicle for our transgene and introduce it into the plant mitochondrial genome, then this mitochondrial plasmid will be more compatible with the plant mitochondrial genome than bacterial plasmid, because its origin would be the mitochondrial genome.

\section{REFERENCES}

Bengtsson BO and Andersson H (1997). The population genetics of plant mitochondrial plasmids. J. Theor. Biol. 188: 163-176.

Borst P and Grivell LA (1971). Mitochondrial ribosomes. FEBS Lett. 13: 73-88.

Esser K, Kuck U, Lang-Hinrichs C, Lemre P, et al. (1986). Plamids of Eukaryotes. Fundamentals and Applications. Springer Verlag, Berlin, 124.

Meinhardt F, Kempken F, Kamper J and Esser K (1990). Linear plasmids among eukaryotes: fundamentals and application. Curr. Genet. 17: 89-95.

Seegers JF, Bron S, Franke CM, Venema G, et al. (1994). The majority of lactococcal plasmids carry a highly related replicon. Microbiology 140: 1291-1300.

Turpen T, Garger SJ, Marks MD and Grill LK (1987). Molecular cloning and physical characterization of a Brassica linear mitochondrial plasmid. Mol. Gen. Genet. 209: 227-233. 